\title{
Editorial
}

\section{Means and Their Inequalities}

\author{
Mowaffaq Hajja, ${ }^{1}$ Peter S. Bullen, ${ }^{2}$ Janusz Matkowski, ${ }^{3}$ \\ Edward Neuman, ${ }^{4}$ and Slavko Simic ${ }^{5}$ \\ ${ }^{1}$ Yarmouk University, Irbid, Jordan \\ ${ }^{2}$ University of British Columbia, 2329 West Mall, Vancouver, BC, Canada V6T $1 Z 4$ \\ ${ }^{3}$ Faculty of Mathematics, Computer Science and Econometrics, University of Zielona Góra, Szafrana 4a, \\ 65-516 Zielona Góra, Poland \\ ${ }^{4}$ Southern Illinois University, 1220 Lincoln Drive, Carbondale, IL 62901, USA \\ ${ }^{5}$ Serbian Academy of Sciences and Arts, Belgrade, Serbia
}

Correspondence should be addressed to Mowaffaq Hajja; mowhajja@yahoo.com

Received 24 June 2013; Accepted 24 June 2013

Copyright (C) 2013 Mowaffaq Hajja et al. This is an open access article distributed under the Creative Commons Attribution License, which permits unrestricted use, distribution, and reproduction in any medium, provided the original work is properly cited.

The theory of means has its roots in the work of the Pythagoreans who introduced the harmonic, geometric, and arithmetic means with reference to their theories of music and arithmetic. Later, Pappus introduced seven other means and gave the well-known elegant geometric proof of the celebrated inequalities among the harmonic, geometric, and arithmetic means.

Nowadays, the families and types of means that are being investigated by researchers and the variety of questions that are being asked about them are beyond the scope of any single survey, with the voluminous book Handbook of Means and Their Inequalities by P. S. Bullen being the best such reference in this direction. The theory of means has grown to occupy a prominent place in mathematics with hundreds of papers on the subject appearing every year.

The strong relations and interactions of the theory of means with the theories of inequalities, functional equations, and probability and statistics add greatly to its importance.

Continuous versions of some means and inequalities among them tie it with real analysis and the theory of integration. The fact that centers of triangles and simplices can be viewed as means of points in the Euclidean spaces makes the subject of interest to geometers.

Positivity and copositivity tests in the theory of forms naturally give rise to questions on internality tests of means arising from forms, making this aspect of the subject of interest to algebraists as well. Extensions of Gauss's outstanding discoveries that relate the evaluation of certain elliptic integrals to iterations of the arithmetic and geometric means that led to the beautiful arithmeticogeometric mean resulted in so many interesting results and lines of research. A quick look at the table of contents of the book Pi and the AGM by J. M. Borwein and P. B. Borwein shows how extensive this line of research is and also shows that the subject is related to almost everything.

The theory of means has applications in so many other diverse fields. Quoting from the preface of the aforementioned book of P. S. Bullen, these include electrostatics, heat conduction, chemistry, and even medicine.

This issue contains several papers that pertain to some of the the aforementioned subjects.

One of the papers is an exposition of certain elementary aspects of the subject, together with several open problems that are within the comprehension of a graduate student. It is hoped that such questions will lead to contributions from experts and amateurs alike. 


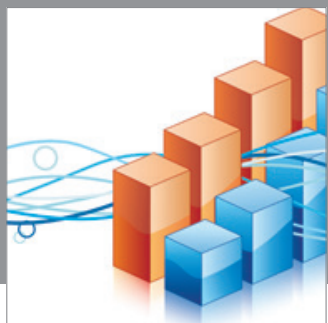

Advances in

Operations Research

mansans

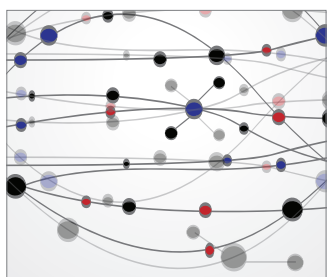

The Scientific World Journal
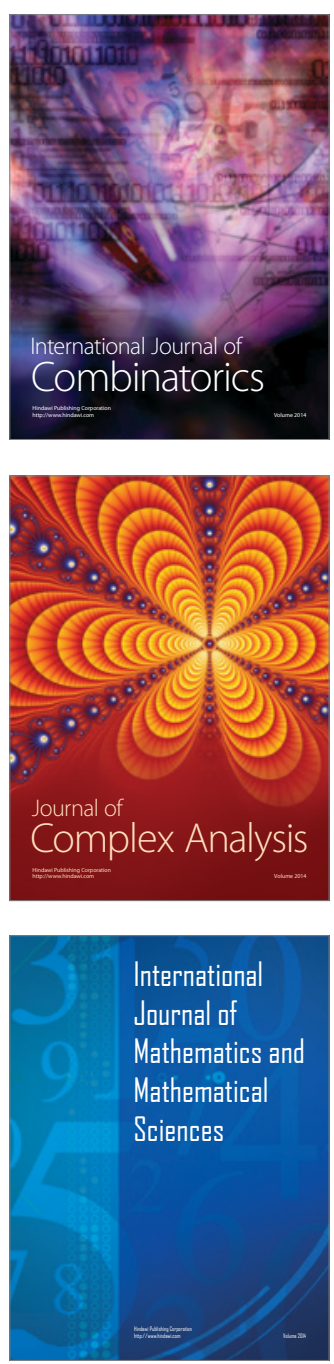
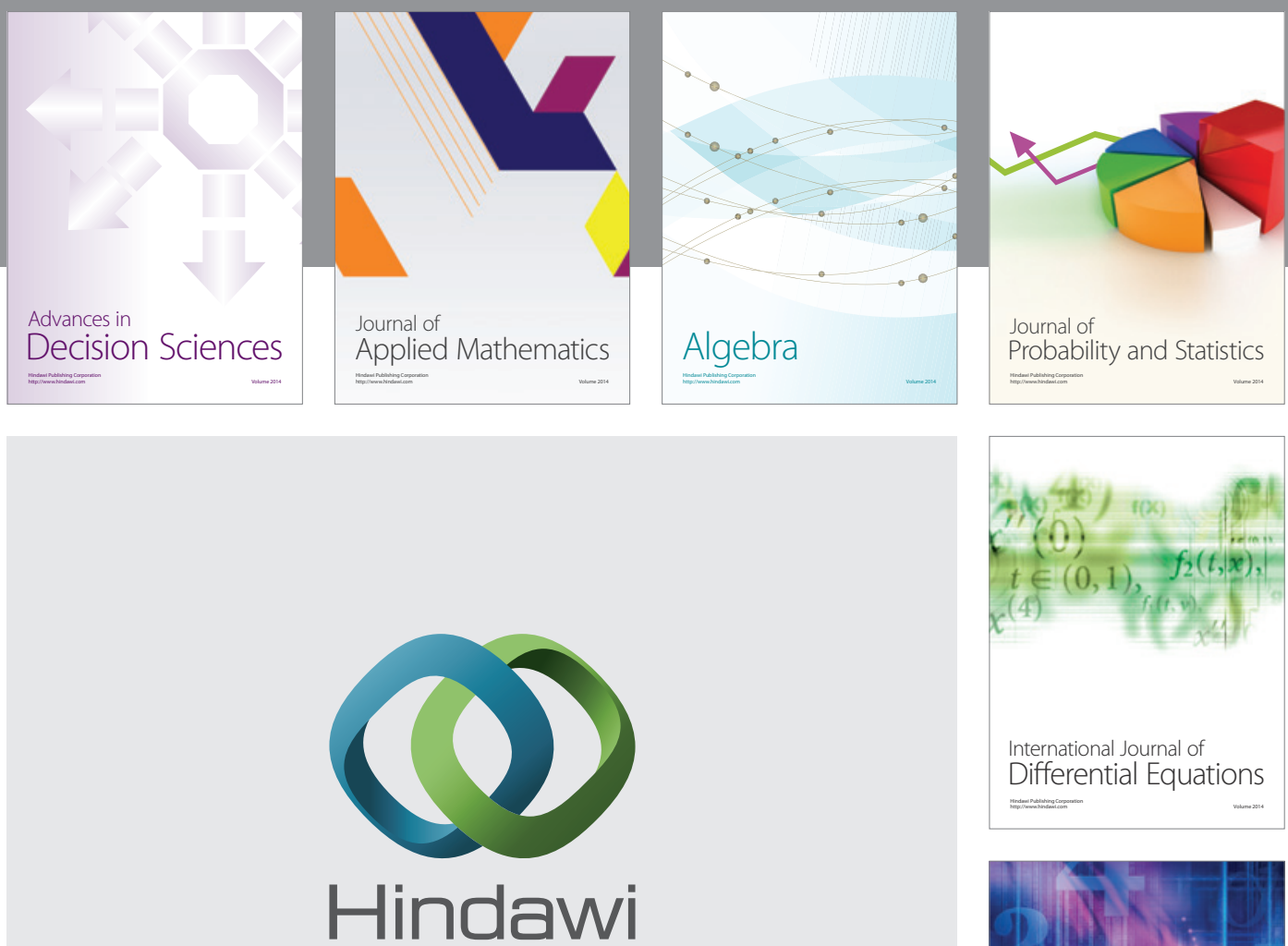

Submit your manuscripts at http://www.hindawi.com
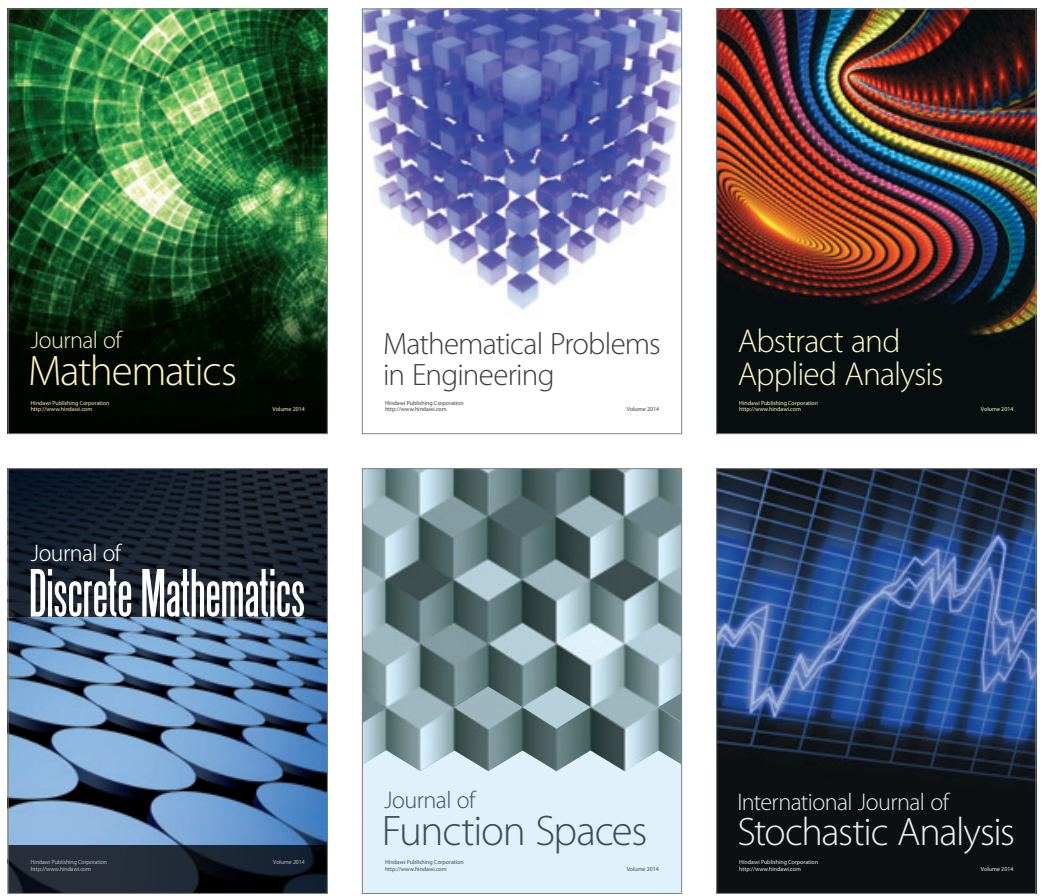

Journal of

Function Spaces

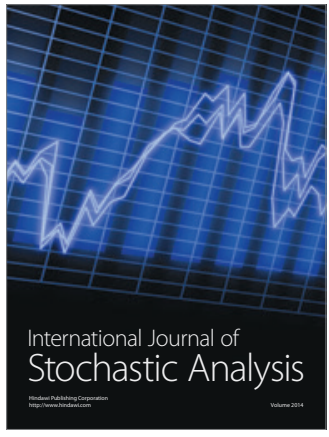

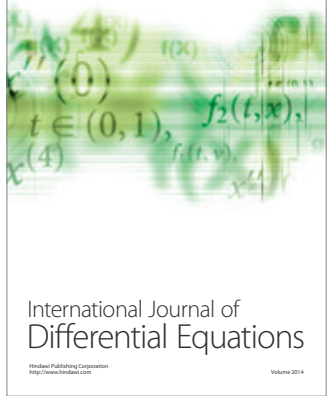
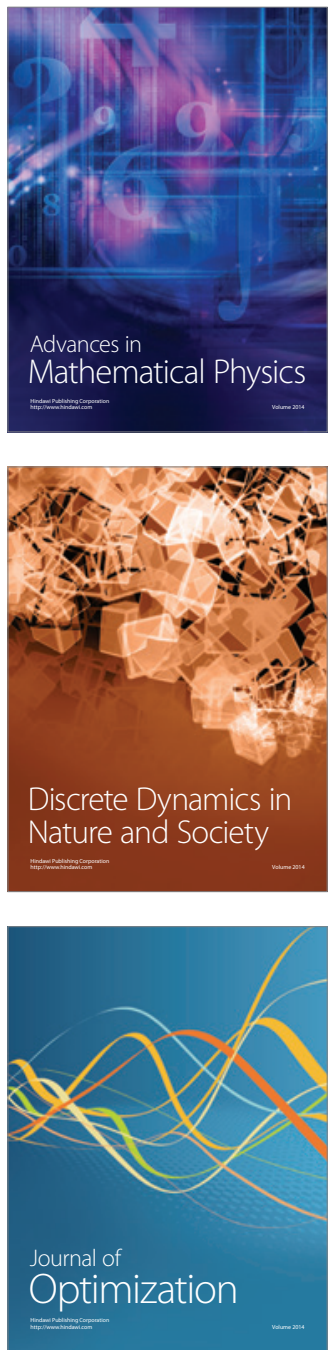